\title{
Selfish elements drive mitochondrial and nuclear genome size in opposite directions
}

\author{
A.A. Mikhaylova ${ }^{1 *}$, A.G. Mikhaylova ${ }^{1}$, K. Ushakova ${ }^{1}$, D. Knorre ${ }^{2}$, I. Mazunin $^{1}$, \\ A. Reymond ${ }^{3}$, K. Gunbin ${ }^{1,4}$, K. Popadin ${ }^{1,3}$ \\ ${ }^{1}$ School of Life Science, Immanuel Kant Federal Baltic University, Kaliningrad, Russia \\ ${ }^{2}$ Belozersky Institute of Physico-Chemical Biology, Moscow State University, Russia \\ ${ }^{3}$ Center for Integrative Genomics, University of Lausanne, Lausanne, Switzerland \\ ${ }^{4}$ Institute of Cytology and Genetics SB RAS, Novosibirsk, Russia \\ *e-mail: mihailovaalina777@yandex.ru
}

Key words: comparative genomics, mitochondrial DNA, repeats, mammals

Motivation and Aim: Nuclear genome size (nucDNA) is higher in long-lived chordata species, where selection against expansion of selfish elements is relaxed due to: (i) lower effective population size (Ne), (ii) higher cell volume, (iii) extended cell cycle period, etc. Mitochondrial genome (mtDNA) has one more, intracellular level of selection, which might change evolutionary forces maintaining the genome size.

Methods and Algorithms: To understand genetic-ecological correlations better we analyzed 840 complete mtDNAs of Mammalia. For each genome we derived dozens of genetic traits (genome length; GC content; abundance, skewness and densities of direct, symmetrical, inverted, complementary and tandem repeats; GC/AT skew etc) and correlated them with several ecological and physiological traits (longevity, body mass, metabolic rate, fecundity etc) using comparative methods like PIC and PGLS.

Results: Analysing 840 complete mitochondrial genomes of mammals species we observed that: (i) genome length variation is explained mainly by variation in control region, which is driven by the abundance of tandem repeats; (ii) tandem repeats are more common in short-lived species; (iii) tandem repeats are AT rich. Putting together all these observations we postulate that mtDNAs of short-lived species are longer and AT-richer as compared to mtDNAs of long-lived species. We explain these observations by two non-mutually exclusive hypotheses: (i) short-lived species have an evolutionary force to maintain fast-replicating mtDNA, i.e. to be more AT rich and to have tandem repeats which can promote fast replication time, simulating or strengthening the origin of replication site [1]; (ii) long-lived species have an evolutionary force to maintain stable mtDNA to decrease somatic mutation rate (high GC content; no repeats which introduce genomic instability) [2]. Since selfish elements propagate more effectively in mtNDA of short-lived species and in nucDNA of long-lived species, we expect and we do observe a negative correlation between the mtDNA size and nucDNA size, emphasizing that opposite forces affects evolution of mtDNA and nucDNA.

Acknowledgements: The study was supported by the 5 Top 100 Russian Academic Excellence Project at the Immanuel Kant Baltic Federal University.

\section{References}

1. Ma H., O'Farrell P.H. (2016) Selfish drive can trump function when animal mitochondrial genomes compete. Nature Genetics. 48(7), 798.

2. Lehmann G., Segal E., Muradian K.K., Fraifeld V.E. (2008). Do mitochondrial DNA and metabolic rate complement each other in determination of the mammalian maximum longevity? Rejuvenation Research. 11(2):409-417. 